\title{
Polymer-Bound 4-Pyridyl-5-hydroxyethyl-thiazole Fluorescent Chemosensors for the Detection of Organophosphate Nerve Agent Simulants
}

Evan P. Lloyd, ${ }^{\dagger}$ Robert S. Pilato, ${ }^{\ddagger}$ and Kelly A. Van Houten ${ }^{*}{ }^{\dagger}$

†Johns Hopkins University Applied Physics Laboratory, 11100 Johns Hopkins Road, Laurel, Maryland 20723, United States

${ }^{\ddagger}$ Lowery Creek Consulting, LLC, 1211 Mill Creek Ln, Kilmarnock, Virginia 22482, United States

Supporting Information

ABSTRACT: Fluorescent sensors have been synthesized for organophosphate nerve agent detection. The resulting 4pyridyl-5-hydroxyethyl structures react with organophosphate nerve agent simulants such as diethylchlorophosphate and diisopropylfluorophosphate and cyclize to form a dihydroquinolizinium ring that results in an increased fluorescence response to long-wave UV excitation. These sensors have been functionalized with monomeric substitutions that allow for covalent incorporation into a polymer matrix for organophosphate detection to develop a fieldable sensor. In addition, inclusion of silicon dioxide into the polymer matrix eliminated false-positive responses from mineral acids, greatly advancing this class of sensors.
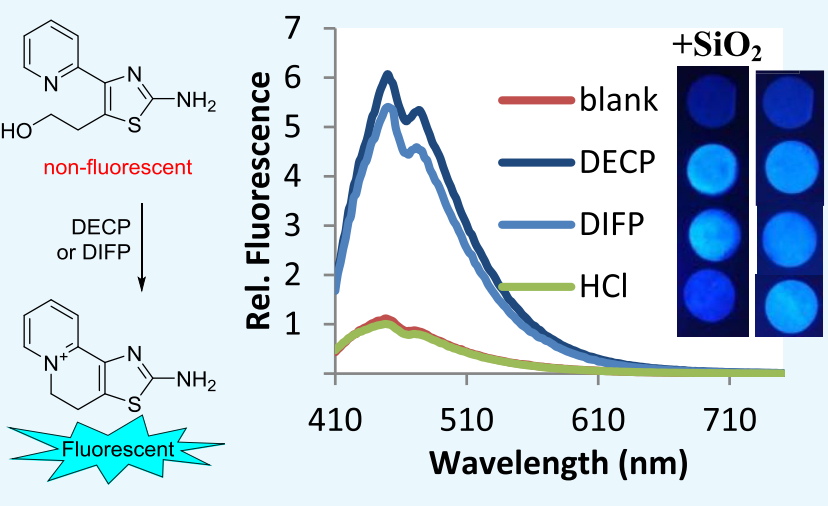

\section{INTRODUCTION}

For more than 70 years, organophosphate acetylcholinesterase inhibitors have been used as weapons of warfare. ${ }^{1-3}$ In addition, this class of compounds are among the most used pesticides. ${ }^{4}$ Given their extensive general use, and the threat posed by the continued use of organophosphate warfare agents, studies to develop methods for the selective detection of these molecules are warranted and timely.,

Recent advances in colorimetric or fluorescent detection using chemical-based sensors include rhodamine-based fluorophores, $^{7-10}$ boron-dipyrromethene dyes, ${ }^{11-15}$ and other similar strategies for the modifications of reactive and highly conjugated small-molecule probes. ${ }^{16-18}$ In some cases, reactivity-based selectivity has been utilized to differentiate between the detection of fluoro- or cyanophosphates. ${ }^{19}$ However, differentiation between common chemicals is difficult and false positives are a major concern in the practical use of fielded sensors. To date, none of these technologies have made their way into the field kits of chemical, biological, radiological, nuclear, and explosives responders. ${ }^{20,21}$

We have worked to improve upon the current state-of-theart through continued study of the 2-pyridyl-1,2-butenyl-4-ol systems. $^{22,23}$ It was demonstrated that pyridines appended to a four-carbon side-chain with a terminal alcohol could be used for the detection of reactive phosphate esters. Restricted rotation and subsequent planarity was achieved in the synthesis of the pseudoaromatic metallo-1,2-enedithiolate 1, providing increased spatial probability of intramolecular coordination of the butanol by the pyridine, resulting in significant fluorescent and absorbance properties (Figure 1). Subsequent work by Swager and co-workers in 2003 demonstrated that these were

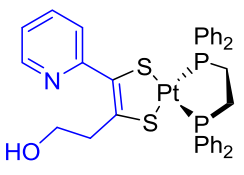

1

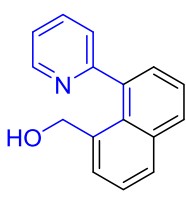

2

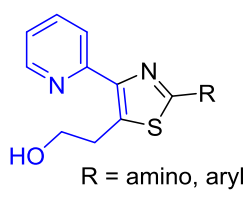

5a-g
Figure 1. Representative fluorescent sensors. The pyridyl-1,2-butenyl4-ol structure is highlighted in blue.

general properties of 2-pyridyl-1,2-butenyl-4-ol systems such as compounds like $2 .^{24,25}$ In 2013, a colorimetric assay using 2pyridyl-1,2-butenyl-4-ol systems to control complexation with iron(II) was explored. ${ }^{26}$ These methods are specific for reactive phosphates, phosphinates, and phosphonates that contain chloro, fluoro, and cyano groups and allow for rapid detection of these esters both colorimetrically and fluorometrically at room temperature.

The following work seeks to implement the above technology toward a robust fieldable sensor. Generation of fully organic and affordable sensors of this class can pave a way toward subsequent immobilization in a polymer matrix to aid

Received: September 7, 2018

Accepted: November 13, 2018

Published: November 27, 2018 
in overcoming problems associated with the acidic byproducts of organophosphate hydrolysis, as has been shown in the literature. $^{14,22,27,28}$

The ethyl-hydroxy group preserves the highly nucleophilic nature toward phosphate esters due to its spatial proximity to the intramolecular pyridine ring. Additionally, a thiazole in conjugation to the pyridine ring is suspected to improve its basicity through resonance stabilization of the protonated form by $2-3 \mathrm{pKa}$ units, resulting in greater reactivity. ${ }^{29}$ Following phosphorylation, the activated phosphate ester is proposed to act as a leaving group for dihydropyrolidinium ring closure as illustrated in Scheme 1. Once the dihydroquinolizinium ring

Scheme 1. Proposed Mechanism for Structures with Increased Fluorescence after Exposure to

Organophosphates

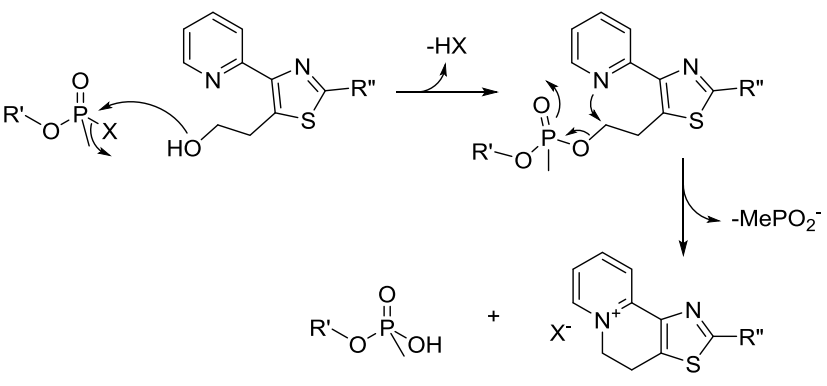

structure is formed, a higher fluorescence yield is observed as a result of increased $\pi$ overlap and forced planarity of the A, B, and $C$ rings. These improvements bring increased pertinence of this strategy in organophosphate detection.

\section{RESULTS AND DISCUSSION}

Synthesis of Thiazole Dyes. Thiazole dyes $\mathbf{5 a}-\mathbf{g}$ can be synthesized from 3-bromo-4-oxo-4-(pyridin-2-yl)butyl acetate, 3, and a thioamide as demonstrated in Scheme 2. Cyclization

\section{Scheme 2. Synthetic Route to Thiazole Sensors}

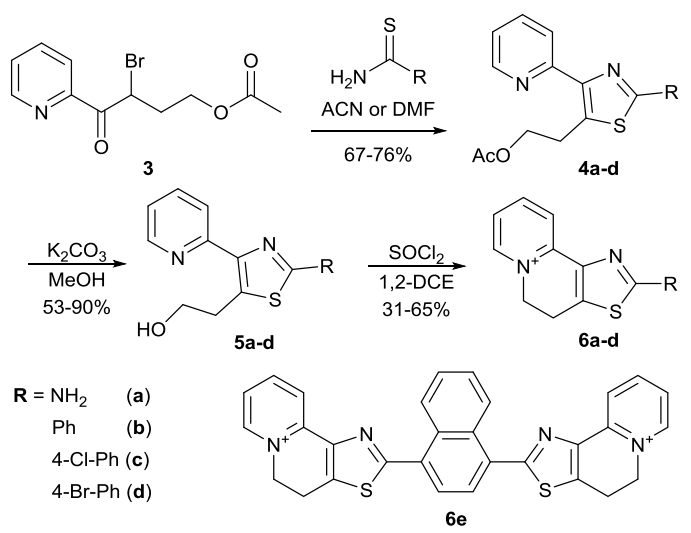

with 4-aryl-thioamides or thiourea gave the target thiazoles in modest yields. After the thiazole formation, facile saponification yields the product in five synthetic steps with an overall yield of $19-38 \%$. In addition to p-substituted phenyl substituents at the thiazole C-2 position, a dimer molecule was made using 1,4-naphthalenedicarbothioamide, and following the same synthetic scheme $6 \mathrm{e}$ was synthesized. Thiazole $4 \mathrm{a}$ was acylated with acrylic acid to provide the monomer 4 f. An

additional monomer unit was prepared via a heck coupling of 4d with ethylene to provide styrene $\mathbf{4 g}$.

The practical utility of organophosphate sensors can be realized by their incorporation into polymer films, which are generated using acryloyl or styryl substituents for radicalinitiated polymerization with styrene. Two of such monomers have been synthesized as shown in Scheme 3. Amino-acid

Scheme 3. Synthesis of Thiazole Monomers

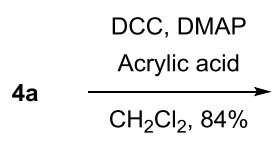

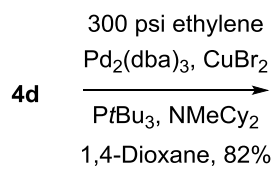

coupling with $N, N^{\prime}$-dicyclohexylcarbodiimide was achieved using acrylic acid to produce the acrylamide $\mathbf{5 b} .^{32}$ The phenyl bromide $\mathbf{5} \mathbf{f}$ is converted to the styryl thiazole $\mathbf{5 g}$ after reaction with ethylene using Heck conditions. ${ }^{33}$

Co-polymers afforded from these monomers limits may provide increased resistance of the sensor to acids and provides precedent for future incorporation into molecularly imprinted polymers (MIPs) to enhance phosphate ester selectivity. ${ }^{34}$ It also allows co-polymerization with additional monomers containing basic functional groups, such as (diisopropylamino)ethyl-methacrylate (DPAEMA). This not only may negate the deleterious effects of the acids in phosphate sensing but also allows for a direct route to preparation of the MIP.

Fluorescent Properties of Thiazole Dyes. Fluorescence emission was expected to increase dramatically after cyclization of the dihydroquinolizinium ring, the results of which for dyes $\mathbf{5 a}-\mathbf{5 g}$ and $\mathbf{6 a}-\mathbf{6 g}$ are summarized in Table 1. For most dyes, the maximum excitation wavelength provided by emission scans was near $350 \mathrm{~nm}$; however, that wavelength cannot be used for emission in testing due to native emission interference by organophosphoryl halides, such as diethylchlorophosphate (DECP) and diisopropylfluorophosphate (DFP), at excitation wavelengths of less than $375 \mathrm{~nm}$. At an excitation wavelength

Table 1. Absorbance and Emissive Properties of Thiazole Sensors

\begin{tabular}{ccccc} 
dye & abs max $\lambda$ & em. $\max \lambda_{395}$ & $\varepsilon^{a}$ & $\phi^{b}(\%)$ \\
\hline 5a & 306 & 449 & 5718 & 0.50 \\
5b & 296 & 450 & 18480 & 3.6 \\
5c & 298 & 449 & 20757 & 2.3 \\
5d & 300 & 453 & 6767 & 2.1 \\
5e & 349 & 440 & 16693 & 11 \\
6a & 380 & 469 & 2201 & 0.31 \\
6b & 360 & 471 & 6767 & 15 \\
6c & 355 & 473 & 5190 & 9.5 \\
6d & 357 & 473 & 4955 & 11 \\
6e & 353 & 459 & 25795 & 4.6
\end{tabular}

${ }^{a}$ Recorded in chloroform at $395 \mathrm{~nm} .{ }^{b}$ Obtained in ethanol at $\lambda_{\text {exit }}=$ $355 \mathrm{~nm}$, relative to 9,10-diphenylanthracene. 
of $395 \mathrm{~nm}, \mathrm{DECP}$ and DFP have no fluorescence emission and a significant emission response from the sensors remained apparent; therefore, $395 \mathrm{~nm}$ was chosen for dye comparison throughout the experiment. Another advantage of choosing an excitation wavelength above $375 \mathrm{~nm}$ is that the precursor alcohols absorb very little light at those wavelengths, resulting in a much reduced emissive response for those starting materials and their $\mathrm{HCl}$ or $\mathrm{HF}$ salts. Synthesis of the dihydroquinolizinium derivatives $\mathbf{6 a}-\mathbf{6 h}$ using thionyl chloride provided purified reference standards for each compound that were characterized alongside their precursor alcohols (Table 1).

Calculation of fluorescent quantum efficiencies in comparison to 9,10-diphenylanthracene as a standard ${ }^{30}$ revealed trends among the thiazole dyes tested. For the 2-aminothiazole 5a, the quantum efficiency did not change dramatically as expected. The rise in fluorescence emission at $\lambda_{\text {exit }}=395 \mathrm{~nm}$ is a direct result of greater absorbance by the fluorophore $\mathbf{6 a}$ at longer wavelengths. For the bis-naphthalene compound 1e, cyclization significantly reduced the quantum yield. For thiazoles $\mathbf{5 b} \mathbf{b} \mathbf{5} \mathbf{d}$, the opposite effect is observed where quantum efficiency is significantly increased after cyclization. The 4-halogen-substituted 2-phenylthiazoles exhibited lower quantum efficiencies in comparison to the 2-phenylthiazole $\mathbf{5 b}$.

The $\pi$-systems of the thiazole and pyridine rings are forced in the same plane upon cyclization, resulting in a maximum absorbance at a longer wavelength for all dyes. As a result, excitation at a wavelength of $395 \mathrm{~nm}$ provides an increase in fluorescence emission intensity, as shown in Figure 2. Quantum efficiencies were obtained for all dihydroquinolizinium derivatives and their respective alcohols. Although quantum efficiencies typically decreased after cyclization, the
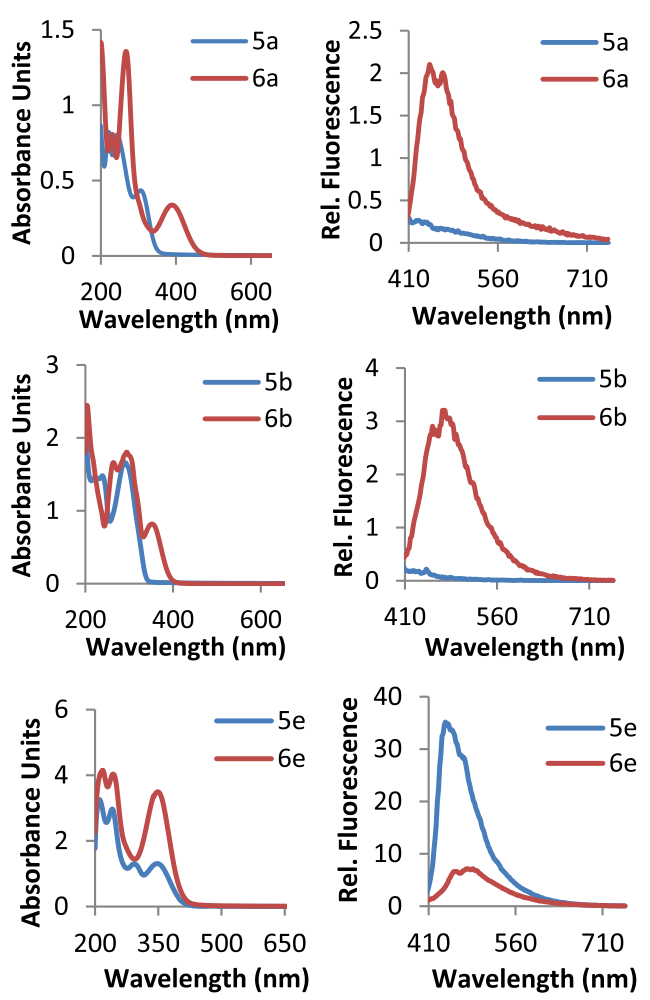

Figure 2. UV absorbance (left) and fluorescence (right) properties of $5 \mathrm{a}$ and $6 \mathrm{a}, 5 \mathrm{~b}$ and $6 \mathrm{~b}$, and $5 \mathrm{e}$ and $6 \mathrm{e}\left(1.0 \times 10^{-6} \mathrm{M}\right)$ in methanol. Fluorescence observed for $\lambda_{\text {exit }}=395$. increase in the absorbance at longer wavelengths provided a striking difference in fluorescence intensity for the $395 \mathrm{~nm}$ excitation wavelength and for the $365 \mathrm{~nm}$ excitation wavelength typical of a longwave UV blacklight.

The increase in $\pi$-conjugation was expected to enhance quantum efficiency, so the opposite observation for $5 e$ and $6 e$ was surprising. A possible explanation is that steric effects cause the 2-phenyl or 2-napthyl structures to be out of the plane of the thiazole $\pi$-system. Molecular mechanics calculations have been used to predict rotational barriers in fluorescence applications, ${ }^{35,36}$ and simple calculations revealed higher conformational energies for structures where the torsion angle between the two rings was fixed at 0 or $180^{\circ}$. Shown in Table 2, the molecular mechanics derived energy difference

Table 2. Minimized Energies of $5 b / 6 b$ and $5 e / 6 e$ Using Conformational Molecular Mechanics ${ }^{a}$

\begin{tabular}{ccccr} 
& energy $(\mathrm{kJ} / \mathrm{mol})$ & $\mathrm{C} 2$ torsion angle $\left(^{\circ}\right)$ & $E^{b b b}$ & $\Delta E^{c}$ \\
\hline $\mathbf{5 b}$ & 302.91 & 10.35 & 302.97 & 0.06 \\
$\mathbf{6 b}$ & 368.22 & -5.7 & 368.22 & 0.00 \\
$\mathbf{5 e}$ & 383.05 & -62.53 & 398.77 & 15.72 \\
$\mathbf{6 e}$ & 450.03 & 61.53 & 464.22 & 14.19
\end{tabular}

${ }^{a}$ Conformer distributions and associated energies were calculated using the MMFF94 model. ${ }^{31}{ }^{b} \mathrm{C}-2$ torsion angle fixed at 0 or $180^{\circ}$. ${ }^{c} \Delta E=\operatorname{EC} 2\left(0,180^{\circ}\right)-\mathrm{EC} 2$ minimized.

between structures with planar constraints and those that are out-of-plane is greater for the bis-thiazole-naphthalene compound $6 \mathrm{e}$ in comparison to the 2-phenyl thiazole structure 6b. Although this is a likely explanation, other factors should be considered as well, such as self-quenching or different excitation states that come into play upon cyclization. 5e was not chosen for further study due to the impracticality of a "turn-off" sensor in comparison to the "turn-on" sensors 5a and $\mathbf{5 b}$.

The newly synthesized thiazole dyes $\mathbf{5 a - 5 e}$ react with organophosphoryl halides, such as organophosphate nerve agent simulants DECP or DFP, as expected. The formation of $\mathbf{6 a}$ and $\mathbf{6 b}$ and subsequent increase in fluorescence emission was examined through the use of DECP in solution phase studies. As a control, hydrochloric acid was also added in the same concentration to show the relative sensitivity to mineral acid. Protonation of $\mathbf{1 a}$ by $\mathrm{HCl}$ results in comparable increase in fluorescence response to DECP after excitation at $365 \mathrm{~nm}$ as shown in Figures 3 and 4. At $395 \mathrm{~nm}$ excitation, a difference

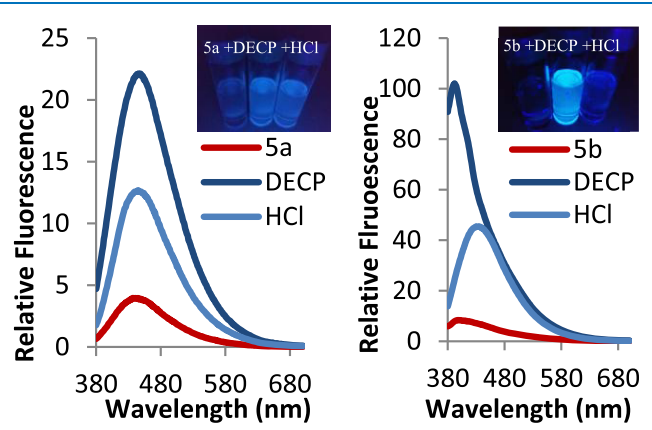

Figure 3. Fluorescence emission titration of $\mathbf{5 a}$ (left) and $\mathbf{5 b}$ (right) in chloroform $\left(1 \times 10^{-4} \mathrm{M}\right)$. Emission is visible after $2 \mathrm{~h}$ exposure to $1 \times 10^{-3} \mathrm{M}$ DIFP or $\mathrm{HCl}\left(\lambda_{\mathrm{ex}}=365 \mathrm{~nm}\right)$. Inset images are respective for fluorescence emission under a long wave UV lamp $\left(\lambda_{\mathrm{ex}}=365 \mathrm{~nm}\right)$. 

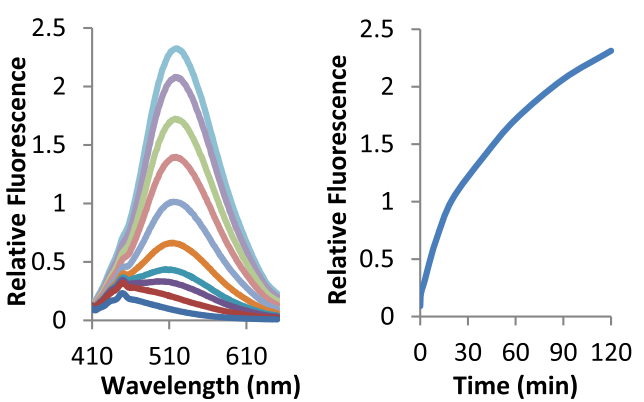

Figure 4. Time course for the fluorescence emission of $5 \mathbf{a}$ in chloroform $\left(10 \times 10^{-6} \mathrm{M}\right)$ with DECP $\left(10 \times 10^{-4} \mathrm{M}\right)$. Left: full emission scans from $T=0$ to $120 \mathrm{~min}$ at $\lambda_{\text {exit }}=395 \mathrm{~nm}$. Right: emission curve at $\lambda_{\max }=515 \mathrm{~nm}$.

between DECP and $\mathrm{HCl}$ is visible due to the increased absorbance of the cyclized product $\mathbf{6} \mathbf{a}$ at longer wavelengths (Supporting Information (SI) Figure 1). The effect of $\mathrm{HCl}$ sensitivity is much reduced for $\mathbf{5 b}$. For practical use in solution phase, $\mathbf{5 b}$ is a better sensor at $365 \mathrm{~nm}$ excitation not only due to greater fluorescence yield upon cyclization but also due to its relative improved contrast of DECP response when compared to the same amount of $\mathrm{HCl}$.

Cyclization was further examined through an increase in fluorescent response over time. In a similar fashion to previous experiments, excess organophosphate was allowed to react with $5 \mathrm{a}$ in a solution over a period of $2 \mathrm{~h}$. The fluorescence response at $\lambda_{\mathrm{ex}}=395 \mathrm{~nm}$ was examined at various time points. Within a few minutes, the presence of organophosphate is evident; however, for complete cyclization to occur, incubation of 5a with DECP must be allowed to take $>2 \mathrm{~h}$ as shown from the solution phase fluorimetry emission experiments. The data support that the reaction is pseudo-first order in nature.

Perhaps a more useful examination of the utility of $\mathbf{5 a}$ and $\mathbf{1 c}$ is through the determination of the limit of detection against organophosphates. Two hours of incubation of $\mathbf{5} \mathbf{a}$ and $\mathbf{5 b}$ was allowed for varying concentrations of DECP, and their fluorescence emission was examined at $\lambda_{\text {exit }}=395 \mathrm{~nm}$. Both dyes exhibit a minimum limit of detection at $1 \times 10^{-4} \mathrm{M}$ for a dye concentration of $1 \times 10^{-4} \mathrm{M}$; roughly 1 equiv of DECP. For 5 equiv DECP, or $1 \times 10^{-4} \mathrm{M} \mathrm{DECP}$, the fluorescence intensity reaches a large jump in intensity. At lower concentrations of the dye, fluorescence intensity begins to have diminishing returns at 1 equiv of organophosphoryl chloride, as a result of slow reaction times and reduced total fluorescence (SI Figure 2).

Synthesis and Characterization of Polystyrene Film Sensors. It remains a concern that mineral acids caused false positives with these sensors in the solution phase. Therefore, the incorporation of the thiazole dyes into polymer films was sought with the hopes that immobilization would deter acid vapor mixing, as well as provide an opportunity for an acid scavenger to be present for increased sensitivity for electrophiles. Previously, organophosphate sensors have been distributed in triethylcitrate/cellulose acetate or RTV118 silicon films that were not optimized to reduce acid sensitivity. $^{22,25}$ However, the incorporation of covalently bonded sensors as part of a polystyrene matrix was a focus of this work.

Two monomers were synthesized for incorporation into a polystyrene film, as shown in Scheme 3. Polymers were made using freshly purified styrene, monomers $\mathbf{5 f}$ or $\mathbf{5 g}$, and catalytic azobisisobutyronitrile (AIBN). To address the acid problem, varying percentages of DPAEMA were added. As a technique for solution phase sensing, a filter paper was coated with $2 \mathrm{~mL}$ of a $20 \%$ polymer $(\mathrm{w} / \mathrm{v})$ in either chloroform or toluene. Once dried, the coated filter paper was cut into $16 \mathrm{~mm}$ diameter circles using a hole punch, and analytes were added to test organophosphoryl halide and organophosphate pesticide detection (SI Figure 3). The DPAEMA-incorporated polymers did not differentiate from false positives as expected. It was not intuitive that $\mathrm{HCl}$ would be visible in all concentrations of basic monomer tested, and despite that, incorporation of the basic monomer proved unhelpful as sensitivity to DECP was decreased in films with higher concentrations (SI Figure 4). These results led to the abandonment of using DPAEMA as a strategy to solve the acid sensitivity issue.

To buffer acid response without the use of an incorporated organic base, silica gel was added to the diluted polymer solution before coating the filter paper to ensure a uniform dispersal as has been shown in other films with $\mathrm{CsCO}_{3} \cdot{ }^{28,37} \mathrm{It}$ was found that silica gel was effective at eliminating the response of the polymer to acid at $1 \mathrm{M}$ concentrations, yet retained the response to DECP and even increased the fluorescence yield of DFP sensing. These results are summarized in Figure 5.

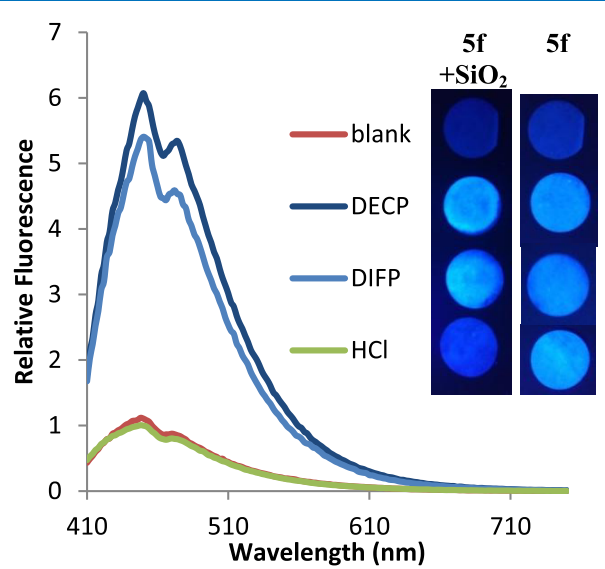

Figure 5. Emission spectra at $\lambda_{\text {exit }}=395 \mathrm{~nm}$ for $\mathrm{SiO}_{2}$ dispersed $0.1 \%$ 5f polystyrene polymer coated on filter paper with DECP, DFP, and $\mathrm{HCl}(1 \mathrm{M})$. Overlaid is the longwave UV fluorescence response at $365 \mathrm{~nm}$ to organophosphate exposure and $1 \mathrm{~N} \mathrm{HCl}$ in methanol on silica-gel-infused filter paper test circles.

To examine vapor-phase organophosphate sensing, the $0.1 \%$ 5f in styrene polymer was spread over a quartz slide and allowed to incubate in a $20 \mathrm{~mL}$ sealed vial that contained $1 \mu \mathrm{L}$ DECP at room temperature. The quartz slide was fitted in a fluorimetry cuvette and examined for fluorescence emission at $395 \mathrm{~nm}$ at 5 and $10 \mathrm{~min}$ time points (SI Figure 5). In similar fashion, the polymer-dispersed filter paper circles were also exposed to DECP and DFP vapors, providing a positive response.

\section{CONCLUSIONS}

These results demonstrate a more practical use of 2-pyridyl1,2-butenyl-4-ol organophosphate sensors than in solution phase. Alleviating acid contamination through the use of dispersed silicon dioxide is a step toward making a robust device that could be used in a variety of applications. The ability to use the sensors as polymer films provides a versatile 
solvent-free platform for both vapor phase and direct droptesting of unknown samples on a test strip to screen for organophosphoryl halide contamination. Further optimization of the polymers, such as use in a MIP, may allow for further differentiation between compounds of interest.

\section{EXPERIMENTAL SECTION}

Materials and General Methods. ${ }^{1} \mathrm{H}$ and ${ }^{13} \mathrm{C}$ NMR spectra were recorded on a Bruker Avance III-HD $400 \mathrm{MHz}$ spectrometer (Bruker, Billerica, MA). ${ }^{13} \mathrm{C}$ NMR spectra were obtained at $100 \mathrm{MHz}$ using a proton-decoupled pulse sequence and are tabulated by observed peak. $\mathrm{CDCl}_{3}$ (Oakwood Chemical, Estill, SC) or $\mathrm{CD}_{3} \mathrm{OD}$ (Sigma-Aldrich, St. Louis, MO) were used as NMR solvents. Chemical shifts $(\delta)$ were reported in parts per million with the residual solvent peak used as an internal standard, $\delta{ }^{1} \mathrm{H} /{ }^{13} \mathrm{C}$ (solvent): 7.26/ $77.00\left(\mathrm{CDCl}_{3}\right) ; 3.30 / 49.00\left(\mathrm{CD}_{3} \mathrm{OD}\right)$; and are tabulated as follows: chemical shift, multiplicity ( $\mathrm{s}$, singlet; $\mathrm{d}$, doublet; $\mathrm{t}$, triplet; $\mathrm{m}$, multiplet; $\mathrm{q}$, quartet), coupling constants, $J$, are reported in hertz $(\mathrm{Hz})$, and number of protons. Highresolution electrospray ionization mass spectra were obtained on a LCQ Orbitrap Velos (ThermoFisher Scientific, Waltham, MA). UV-vis spectra were recorded at room temperature on a Cary 5000 UV-vis-NIR spectrophotometer (Varian, Palo Alto, CA). Solution phase emission and excitation spectra were obtained at room temperature using a Spex FluoroMax-2 Spectrofluorometer (Horiba Scientific, Edison, NJ). Solidphase emission and excitation spectra were obtained at room temperature using an Infinite M1000 microplate reader (Tecan, Morrisville, NC) using 24-well corning cell culture plates painted with black with matte low-reflectance camouflage spray paint. Thin-layer chromatography was performed on ready-to-use glass-backed silica gel 60 plates (SigmaAldrich, St. Louis, MO). Flash column chromatography was performed over Acros silica gel (40-60 $\mu \mathrm{m}, 200-400$ mesh). All the reactions were performed under an inert atmosphere of either Zero-grade nitrogen or argon (Airgas, Radnor, PA). All the chemicals were obtained from Sigma-Aldrich (St. Louis, MO), Fisher Scientific (Pittsburgh, PA), or Oakwood Chemicals (Estill, SC) and used without further purification.

Synthesis of Compounds $1 \mathbf{a}-\mathbf{g}-\mathbf{6 a}-\mathbf{g}$. 1-(2-Pyridyl)-2bromo-4-acetoxybutan-1-one 3 was prepared according to the literature procedure. ${ }^{22}$ Further details and spectral data for the synthesis of these analogues and their synthetic intermediates are provided in the Supporting Information.

General Procedure for the Polymerization of Polystyrene Films. To benzene $(5 \mathrm{~mL})$ in a $30 \mathrm{~mL}$ vial fitted with a stir bar was added $2 \mathrm{~mL}$ styrene that had been passed through a column of inhibitor remover resin. To this was added monomers $4 \mathbf{a}$ or $4 \mathrm{e}$ with $0,1,5$, or $10 \% \mathrm{v} / \mathrm{v}$ (diisopropylamino)ethyl-methacrylate (DPAEMA). Finally, a catalytic amount of azobisisobutyronitrile (AIBN) was added. The filled vial was fitted to a Schlenk manifold and placed under an atmosphere of argon. The reaction mixture was cooled in liquid nitrogen, evacuated with vacuum and warmed under static vacuum to degas the solution. This freeze-pumpthaw process was completed twice more before the vial was sealed under gentle heating of the neck with a propane torch under static vacuum. The sealed vial was stirred and heated to $75{ }^{\circ} \mathrm{C}$ for $16 \mathrm{~h}$. After this time, polymerization was complete and the film cast and dried.

Analysis of Solution Phase Fluorimetry Response of Thiazole Dyes to Organophosphates. Thiazoles 5a, 5b, $\mathbf{5 c}$, 5d, and $\mathbf{5 e}$ were analyzed for their fluorescence emission after incubation with organophosphate nerve agent simulants diethylchlorophosphate (DECP) or diisopropylfluorophosphate (DFP). The dyes were dissolved in either methanol or chloroform to a final concentration of $1 \times 10^{-6}$ or $1 \times 10^{-7} \mathrm{M}$, respectively. Three milliliters of diluted solution was pipetted into a $1.0 \times 1.0 \mathrm{~cm}^{2}$ quartz fluorimetry cell with clear walls on all four sides, capped, and the cuvette was placed into the Fluoromax-2 spectrophotometer and the door fully sealed. Analysis was performed at either 355 or $395 \mathrm{~nm}$ excitation wavelengths, and the emission spectra were scanned from 410 to $650 \mathrm{~nm}$ at a rate of 5 scans per second. The spectral bandpass was set to $5 \mathrm{~nm}$ for both the excitation and emission. A desired amount of simulant is added directly to the cuvette for additional testing, and a stir bar is fitted to the cuvette for facile mixing on an external magnetic stir plate. The polymer films were analyzed for their base and postexposure response by coating a small flat quartz insert with polystyrene films by drop-casting using a concentrated suspension of polymer in chloroform. The insert was placed at a $45^{\circ}$ angle to the excitation beam, allowing for the detection of the fluorescence emission by the detector.

Production of Polymer Film-Coated Filter Paper Test Strips and Testing. Polystyrene films were concentrated to dryness in vacuo and suspended in chloroform $(5 \mathrm{~mL} / \mathrm{g})$. Silica gel, alumina, or fumed silica was suspended into the solution $(50 \mathrm{mg} / 2 \mathrm{~mL})$. The films were coated onto $85 \mathrm{~mm}$ diameter qualitative fast-flow filter paper $(2 \mathrm{~mL}$ solution $)$ and allowed to dry. For uniformity, a $16 \mathrm{~mm}$ diameter circular hole-punch was used to cut out circular filter paper samples. These test circles were exposed to analytes directly. For complete coating, at least $40 \mu \mathrm{L}$ was used to produce a uniform fluorescent response. Once dried, the test circles were placed into the bottom of a well in a matte black-painted 24-well microplate and analyzed for their fluorescent response at $\lambda_{\text {exit }}=$ 395 using a microplate reader. Analysis was performed at either 355 or $395 \mathrm{~nm}$ excitation wavelengths, and the emission spectra were scanned from 365 to 750 or 410 to $750 \mathrm{~nm}$, with a scan frequency of every $2 \mathrm{~nm}$. The excitation band width was set to $5 \mathrm{~nm}$. Gain was optimized for the well that contained the highest expected fluorescence to be analyzed.

Calculation of Quantum Yields. Quantum yields in ethanol were calculated from a standard curve for samples at concentrations of $0.2 \times 10^{-5}, 0.4 \times 10^{-5}, 0.6 \times 10^{-5}, 0.8 \times$ $10^{-5}$, and $1.0 \times 10^{-5} \mathrm{M}$ for $5 \mathbf{5 a}, \mathbf{6 a}, \mathbf{5 b}, \mathbf{5 c}$, and $5 \mathrm{~d}$, and $0.2 \times$ $10^{-6}, 0.4 \times 10^{-6}, 0.6 \times 10^{-6}, 0.8 \times 10^{-6}$, and $1.0 \times 10^{-6} \mathrm{M}$ for 6b, 6c, 6d, 5e and 6e, respectively. A volume of $200 \mu \mathrm{L}$ was placed in each respective well in a 96-well clear or black polystyrene flat-bottom microplate (Corning, Tewksbury, $\mathrm{MA}$ ). Both the absorbance at $355 \mathrm{~nm}$ and the total integrated fluorescence at $\lambda_{\text {exit }}=355$ were plotted for each compound. Using the literature quantum efficiency value of 0.95 for 9,10diphenylanthracene in ethanol, ${ }^{30}$ the resulting slopes from fluorescence over absorbance were compared to that generated for the fluorescence standard at concentrations of $0.2 \times 10^{-7}$, $0.4 \times 10^{-7}, 0.6 \times 10^{-7}, 0.8 \times 10^{-7}$, and $1.0 \times 10^{-7} \mathrm{M}$. Total integrated fluorescence was calculated using the following formula for integration of a manual plot: $\int_{a}^{b} y(x) \mathrm{d} x=\frac{(b-a)}{N} \sum_{a}^{b} y(x)$, where $a=365 \mathrm{~nm}, b=749$ $\mathrm{nm}$, and $N=192$.

Computational Details. Molecular mechanics (MMFF94) calculations ${ }^{31}$ were performed using Spartan'16 
V2.07 (Wavefunction, Irvine, CA). Conformational structures for $\mathbf{5 b}, \mathbf{5 e}, \mathbf{6 b}$, and $\mathbf{6 e}$ were minimized using this function and associated energies calculated and recorded for the minimumenergy conformation. For these structures, the torsion angle was examined between $\mathrm{C} 2$ of the thiazole ring and the phenyl ring of $5 b$ and $6 b$ or napthyl ring of $5 e$ and $6 e$, respectively (Table 2). The calculations were repeated for each structure after constraining this torsion angle at 0 or $180^{\circ}$, and the respective energies are tabulated for comparison.

\section{ASSOCIATED CONTENT}

\section{S Supporting Information}

The Supporting Information is available free of charge on the ACS Publications website at DOI: 10.1021/acsomega.8b02313.

Experimental procedures and characterization; synthesis of polystyrene films; procedure for filter paper coating, exposure and measurements; NMR spectra for synthesized compounds (PDF)

\section{AUTHOR INFORMATION}

\section{Corresponding Author}

*E-mail: Kelly.Van.Houten@jhuapl.edu. Tel: 240-228-2094.

\section{ORCID $\odot$}

Evan P. Lloyd: 0000-0001-6984-7400

\section{Author Contributions}

E.P.L. and K.A.V.H. designed the experiments, performed the chemical synthesis, characterization, and analysis, and wrote the manuscript. R.S.P. designed the experiments, performed the chemical synthesis, and reviewed the manuscript.

\section{Funding}

The Johns Hopkins University Applied Physics Laboratory internally funded this research.

\section{Notes}

The authors declare no competing financial interest.

\section{ACKNOWLEDGMENTS}

We would like to thank Dr. James K. Johnson for insightful discussions and invaluable help in editing this manuscript and Daniel Van Buren for his help in compiling samples and characterizations.

\section{ABBREVIATIONS}

DPAEMA, (diisopropylamino)ethyl-methacrylate; AIBN, azobisisobutyronitrile; DECP, diethylchlorophosphate; DFP, diisopropylfluorophosphate; MIP, molecularly imprinted polymer

\section{REFERENCES}

(1) Somani, S. M. Chemical Warfare Agents, 1st ed.; Academic Press: London, 1992.

(2) Sidell, F. R.; Borak, J. Chemical Warfare Agents: II. Nerve Agents. Ann. Emerg. Med. 1992, 21, 865-871.

(3) Quinn, D. M. Enzymes of the Cholinesterase Family, 5th ed.; Plenum Press: Madras, India, 1994.

(4) Khan, S. U. Pesticides in the Soil Environment, 1st ed.; Wakeman, R. J., Ed.; Elsevier Scientific Pub. Co., 1980.

(5) Kim, K.; Tsay, O. G.; Atwood, D. A.; Churchill, D. G. Destruction and Detection of Chemical Warfare Agents. Chem. Rev. 2011, 111, 5345-5403.
(6) Burnworth, M.; Rowan, S. J.; Weder, C. Fluorescent Sensors for the Detection of Chemical Warfare Agents. Chem. - Eur. J. 2007, 13, 7828-7836.

(7) Goswami, S.; Manna, A.; Paul, S. Rapid "naked Eye" Response of DCP, a Nerve Agent Simulant: From Molecules to Low-Cost Devices for Both Liquid and Vapour Phase Detection. RSC Adv. 2014, 4, 21984-21988.

(8) Weerasinghe, A. J.; Schmiesing, C.; Sinn, E. Synthesis, Characterization, and Evaluation of Rhodamine Based Sensors for Nerve Gas Mimics. Tetrahedron 2011, 67, 2833-2838.

(9) $\mathrm{Wu}, \mathrm{X} . ; \mathrm{Wu}, \mathrm{Z}$; $\mathrm{Han}, \mathrm{S}$. Chromogenic and Fluorogenic Detection of a Nerve Agent Simulant with a Rhodamine-Deoxylactam Based Sensor. Chem. Commun. 2011, 47, 11468.

(10) Wu, W. H.; Dong, J.; Wang, X.; Li, J.; Sui, S.; Chen, G.; Liu, J.; Zhang, M. Fluorogenic and Chromogenic Probe for Rapid Detection of a Nerve Agent Simulant DCP. Analyst 2012, 137, 3224.

(11) Climent, E.; Biyikal, M.; Gawlitza, K.; Dropa, T.; Urban, M.; Costero, A. M.; Martínez-Máñez, R.; Rurack, K. A Rapid and Sensitive Strip-Based Quick Test for Nerve Agents Tabun, Sarin, and Soman Using BODIPY-Modified Silica Materials. Chem. - Eur. J. 2016, 22, $11138-11142$.

(12) Xia, H. C.; Xu, X. H.; Song, Q. H. BODIPY-Based Fluorescent Sensor for the Recognization of Phosgene in Solutions and in Gas Phase. Anal. Chem. 2017, 89, 4192-4197.

(13) Gotor, R.; Gaviña, P.; Ochando, L. E.; Chulvi, K.; Lorente, A.; Martínez-Máñez, R.; Costero, A. M. BODIPY Dyes Functionalized with 2-(2-Dimethylaminophenyl)ethanol Moieties as Selective OFFON Fluorescent Chemodosimeters for the Nerve Agent Mimics DCNP and DFP. RSC Adv. 2014, 4, 15975-15982.

(14) Barba-Bon, A.; Costero, A. M.; Gil, S.; Harriman, A.; Sancenón, F. Highly Selective Detection of Nerve-Agent Simulants with BODIPY Dyes. Chem. - Eur. J. 2014, 20, 6339-6347.

(15) Fu, G.-L.; Pan, H.; Zhao, Y.-H.; Zhao, C.-H. Solid-State Emissive Triarylborane-Based BODIPY Dyes: Photophysical Properties and Fluorescent Sensing for Fluoride and Cyanide Ions. Org. Biomol. Chem. 2011, 9, 8141.

(16) Mahapatra, A. K.; Maiti, K.; Sahoo, P.; Nandi, P. K. A New Colorimetric and Fluorescent Bis(coumarin)methylene Probe for Fluoride Ion Detection Based on the Proton Transfer Signaling Mode. J. Lumin. 2013, 143, 349-354.

(17) Ajami, D.; Rebek, J. Chemical Approaches for Detection and Destruction of Nerve Agents. Org. Biomol. Chem. 2013, 11, 3936.

(18) Royo, S.; Gotor, R.; Costero, A. M.; Parra, M.; Gil, S.; Martínez-Máñez, R.; Sancenón, F. Aryl Carbinols as Nerve Agent Probes. Influence of the Conjugation on the Sensing Properties. New J. Chem. 2012, 36, 1485.

(19) Royo, S.; Costero, A. M.; Parra, M.; Gil, S.; Martínez-Máñez, R.; Sancenón, F. Chromogenic, Specific Detection of the Nerve-Agent Mimic DCNP (a Tabun Mimic). Chem. - Eur. J. 2011, 17, 69316934.

(20) Sferopoulos, R. A Review of Chemical Warfare Agent (CWA) Detector Technologies and Commercial-Off-The-Shelf Items; Australian Government Department of Defence, 2009; pp 1-98.

(21) Kangas, M. J.; Burks, R. M.; Atwater, J.; Lukowicz, R. M.; Williams, P.; Holmes, A. E. Colorimetric Sensor Arrays for the Detection and Identification of Chemical Weapons and Explosives. Crit. Rev. Anal. Chem. 2017, 47, 138-153.

(22) Van Houten, K. A.; Heath, D. C.; Barringer, C. A.; Rheingold, A. L.; Pilato, R. S. Functionalized 2-Pyridyl-Substituted Metallo-1,2Enedithiolates. Synthesis, Characterization, and Photophysical Properties of (dppe) $\mathrm{M}\left\{\mathrm{S} 2 \mathrm{C} 2(2\right.$-pyridine (ium) $\left.)\left(\mathrm{CH} 2 \mathrm{CH} 2 \mathrm{OR}^{\prime \prime}\right)\right\}$ and (dppe) $\mathrm{M}[\{\mathrm{S} 2 \mathrm{C} 2(\mathrm{CH} 2 \mathrm{CH} 2-\mathrm{N}-2-$ Pyridinium $)\}]+\left(\mathrm{R}^{\prime \prime}=\mathrm{H}\right.$, Acetyl, Lauroyl; M = Pd, Pt; Dpp. Inorg. Chem. 1998, 37, 4647-4653.

(23) Van Houten, K. A.; Heath, D. C.; Pilato, R. S. Rapid Luminescent Detection of Phosphate Esters in Solution and the Gas Phase Using (dppe) $\mathrm{Pt}\{\mathrm{S} 2 \mathrm{C} 2$ (2-pyridyl) $(\mathrm{CH} 2 \mathrm{CH} 2 \mathrm{OH})\}$ [5]. J. Am. Chem. Soc. 1998, 120, 12359-12360.

(24) Van Houten, K. A.; Heath, D. C.; Pilato, R. S. In Luminescent Detection of Activated Phosphate Esters in Solution and in the Gas 
Phase, Presented at the 216th National Meeting of the American Chemical Society, Boston, MA, 1998.

(25) Zhang, S. W.; Swager, T. M. Fluorescent Detection of Chemical Warfare Agents: Functional Group Specific Ratiometric Chemosensors. J. Am. Chem. Soc. 2003, 125, 3420-3421.

(26) Ordronneau, L.; Carella, A.; Pohanka, M.; Simonato, J.-P. Chromogenic Detection of Sarin by Discolouring Decomplexation of a Metal Coordination Complex. Chem. Commun. 2013, 49, 89468948.

(27) Cai, Y.-C.; Li, C.; Song, Q.-H. Fluorescent Chemosensors with Varying Degrees of Intramolecular Charge Transfer for Detection of a Nerve Agent Mimic in Solutions and in Vapor. ACS Sens. 2017, 2, 834-841.

(28) Gotor, R.; Costero, A. M.; Gil, S.; Parra, M.; Martínez-Máñez, R.; Sancenón, F. A Molecular Probe for the Highly Selective Chromogenic Detection of DFP, a Mimic of Sarin and Soman Nerve Agents. Chem. - Eur. J. 2011, 17, 11994-11997.

(29) Kaiwar, S. P.; Hsu, J. K.; Liable-Sands, L. M.; Rheingold, A. L.; Pilato, R. S. Synthesis and Properties of Heterocyclic Substituted 1,2Enedithiolates of Nickel, Palladium, and Platinum Inorg. Chem. 1997, 4234 DOI: 10.1021/ic970194s.

(30) Morris, J. V.; Mahaney, M. A.; Huber, J. R. Fluorescence quantum yield determinations. 9,10-Diphenylanthracene as a reference standard in different solvents. J. Phys. Chem. 1976, 80, 969.

(31) Halgren, T. A. Merck Molecular Force Field. I. Basis, Form, Scope, Parameterization, and Performance of MMFF94. J. Comput. Chem. 1996, 17, 490-519.

(32) El-Hamouly, S. H.; Azab, M. M.; Diab, A. S. M.; Hegazy, M. A. Synthesis and Thermal Characteristics of N-(Thiazol-2-Y1)methacrylamide-Acrylonitrile Copolymers. Asian J. Chem. 2012, 24, 176-180.

(33) Southard, G. E.; Van Houten, K. A.; Murray, G. M. Heck Cross-Coupling for Synthesizing Metal-Complexing Monomers. Synthesis 2006, 2475-2477.

(34) Southard, G. E.; Van Houten, K. A.; Murray, G. M. Soluble and Processable Phosphonate Sensing Star Molecularly Imprinted Polymers. Macromolecules 2007, 40, 1395-1400.

(35) Sadrai, M.; Hadel, L.; Sauers, R. R.; Husain, S.; KroghJespersen, K.; Westbrook, J. D.; Bird, G. R. Lasing Action in a Family of Perylene Derivatives: Singlet Absorption and Emission Spectra, Triplet Absorption and Oxygen Quenching Constants, and Molecular Mechanics and Semiempirical Molecular Orbital Calculations. J. Phys. Chem. 1992, 96, 7988-7996.

(36) Pasco, S. T.; Baker, G. L. Rotational Barriers and Conjugation in Polyphenylene Oligomers. Synth. Met. 1997, 84, 275-276.

(37) El Sayed, S.; Pascual, L.; Agostini, A.; Martínez-Máñez, R.; Sancenõn, F.; Costero, A. M.; Parra, M.; Gil, S. A Chromogenic Probe for the Selective Recognition of Sarin and Soman Mimic DFP. ChemistryOpen 2014, 3, 142-145. 